\title{
Numerical study for anisotropic influences on elastic wavefields near surface
}

\author{
${\text { Rina } \text { YONEKI }^{1} \text {, Hitoshi MIKADA }}^{1}$ and Junichi TAKEKAWA ${ }^{1}$ \\ ${ }^{1}$ Dept. of Civil and Earth Res. Eng., Kyoto University
}

\begin{abstract}
We think anisotropic velocity analysis, which is known important for understanding the behavior of hydraulically generated fractures and due to stress surrounded a borehole, would be key to understand the state consolidation of sediments near the surface. There are many studies on seismic wave propagation in transversely isotropic and orthorhombic media. In the most of those studies, the magnitude of anisotropy is assumed to be weak. In addition, there are few studies on seismic wavefields in quite strongly anisotropic media. Therefore, it may not be appropriate to apply their theories directly to strongly anisotropic subsurface media. It is necessary to understand the effects of the anisotropy on the behavior of seismic wave propagation in strongly anisotropic media in the seismic exploration. In this study, we investigate the influence of strong anisotropy on received seismic waveforms using three-dimensional numerical models, and verified capability of detecting subsurface anisotropy. Our numerical models contain an isotropic and an anisotropic (transversely isotropic) layer in an isotropic background subsurface. Since the difference between the two models is only the anisotropy in the vertical propagation velocity, we could observe the influence of anisotropy in the residual wavefield that is the difference in the observed wavefields of two models. The residual waveforms could be exploited to estimate both the order of anisotropy and the thickness of anisotropic layer in subsurface.
\end{abstract}

\section{INTRODUCTION}

Anisotropic velocity analysis has been wide used for understanding how hydraulic induced fractures could propagate from boreholes due to local stress orientations. Seismic anisotropy represents the orientation, the difference between the maximum and the minimum stress components, which could reflect the consolidation history of the materials after sedimentation. Recent development of unconventional hydrocarbon reservoirs has revealed that subsurface materials are much anisotropic than expected ${ }^{1)}$. Materials have anisotropy due to crustal stress and arranged direction of rock fractures, layering structure of sediments, alternating layers. There are many studies on seismic wave propagation in transversely isotropic and orthorhombic media ${ }^{2), 3)}$. In most of those studies, the magnitude of anisotropy is assumed to be weak. In addition, there are few studies on seismic wavefields in quite strongly anisotropic media.

In this study, to obtain detailed information of near surface anisotropy, we investigate the influence of strong anisotropy on received seismic waveforms with numerical simulations using finite-difference method (FDM). We focus on P-S converted waves as signals especially affected by strong anisotropy in seismic data. In general, natural earthquake seismic data have signals between initial P-wave and initial S-wave, and they are explained by existence of inhomogeneous media ${ }^{4}$. However, we hypothesis that these signals between initial P-wave and initial S-wave are observed because of not only inhomogeneity but also P-S converted waves due to subsurface strong anisotropy. Our numerical models have isotropic and anisotropic layers in homogeneous medium. Comparing the received waveforms of two models, we verified capability of detecting subsurface anisotropy.

\section{SIMULATION METHODS}

\section{(1) Governing equations}

The equation of motion ${ }^{5}$ and the constitutive relationship (Hook's low) are given as equation (1) and (2), respectively.

$$
\begin{aligned}
\rho \ddot{u}_{i} & =\tau_{i j, j}+f_{i}, \\
\tau_{i j} & =c_{i j, k l} \varepsilon_{k l},
\end{aligned}
$$

In equation (1), $u_{i}$ is particle displacement vector, $\tau_{i j, j}$ is stress tensor, $f_{j}$ is body force that represents source and $\rho$ is density. In equation (2), $c_{i j, k l}$ is stiffness tensor and $\varepsilon_{k l}$ is strain tensor. 
Then, equation (1) and (2) can be rewritten as,

$$
\begin{gathered}
\rho \dot{v}_{i}=\tau_{i j, j}+f_{i}, \\
\dot{\tau}_{i j}=c_{i j, k l} v_{k, l} .
\end{gathered}
$$

where, $v$ is velocity. We use the velocity-stress FD scheme to solve equations (4) and (5).

We use VTI medium (transversally isotropic media with a vertical axis of symmetry) as an anisotropic medium in this study. VTI medium has isotropic phase velocity in horizontal plane and has a symmetry axis of velocity in vertical direction. Equation (6) shows equation (5) rewritten for VTI medium:

$$
\left(\begin{array}{l}
\dot{\tau}_{x x} \\
\dot{\tau}_{y y} \\
\dot{\tau}_{z z} \\
\dot{\tau}_{y z} \\
\dot{\tau}_{z x} \\
\dot{\tau}_{x y}
\end{array}\right)=\left(\begin{array}{cccccc}
c_{11} & c_{12} & c_{13} & 0 & 0 & 0 \\
& c_{11} & c_{13} & 0 & 0 & 0 \\
& & c_{33} & 0 & 0 & 0 \\
& & & c_{44} & 0 & 0 \\
& s y m . & & & c_{44} & 0 \\
& & & & & c_{66}
\end{array}\right)\left(\begin{array}{c}
v_{x, x} \\
v_{y, y} \\
v_{z, z} \\
v_{y, z}+v_{z, y} \\
v_{z, x}+v_{x, z} \\
v_{x, y}+v_{y, x}
\end{array}\right)
$$

Note that $c_{12}$ can be expressed by other values as $c_{12}=c_{11}-2 c_{66}$. This means that stiffness matrix of VTI has only five independent components among twelve non-zero components.

\section{(2) Finite-difference scheme}

To simulate seismic wave propagation, we have implemented a FDM. For calculating elastic wave propagation using FD scheme, standard staggered gird scheme ${ }^{6)}$ is well known. However, when a model has lower symmetry anisotropy than orthorhombic or has large impedance contrast, staggered grid scheme increases numerical error. That is because components of velocity $v_{i}$ and stress $\tau_{i j}$ in standard staggered grid scheme are not defined at the same location respectively. Because of this reason, we use rotated staggered grid for FD scheme ${ }^{7)}{ }^{8)}$. In rotated staggered grid scheme, all components of $v_{i}$ and $\tau_{i j}$ are defined at the same location, there is no need to interpolate velocity and $c_{i j, k l}$. So it can be applied to general anisotropic media. Additionally, it becomes possible to calculate seismic wave propagation without explicit boundary condition at high contrast interfaces.

\section{NUMERICAL MODELS}

We conducted numerical experiments using two different inhomogeneous models. Each model is horizontally layered model and includes an isotropic or anisotropic layer as a velocity anomaly in an isotropic homogeneous medium.

Fig. 1 shows the two numerical models. We call the models of Fig. 1 (a) and (b) as the isotropic anomaly model and the anisotropic anomaly model, respectively. The top layer of Fig. 1 (a) is an isotropic medium and that of Fig. 1 (b) is a VTI medium. The thickness of top layers is $600 \mathrm{~m}$ and the depth of the source from the surface is $1291 \mathrm{~m}$. The source function is ricker wavelet with a dominant frequency $15 \mathrm{~Hz}$ and the excitation direction is vertical direction (z-direction). We implement CPML boundary condition ${ }^{9}$ for five edge faces except the top surface. The vertical velocities of top layers in these two models are the same. The horizontal velocity of anisotropic medium in Fig. 1 (b) is larger than the vertical velocity and the degree of anisotropy, i.e. the ratio of maximum phase velocity and minimum phase velocity, is $30 \%\left(\mathrm{~V}_{x, y} / \mathrm{V}_{z}=1.3\right)$. Stiffness components in equation (6) of VTI medium are following; $c_{11}=12.10 \mathrm{GPa}, c_{33}=20.45 \mathrm{GPa}, c_{13}$ $=2.847 \mathrm{GPa}, c_{44}=8.281 \mathrm{GPa}, c_{66}=4.900 \mathrm{GP}$. The bottom media of two models have the same physical property. Table 1 shows the vertical phase velocities and density of the models.

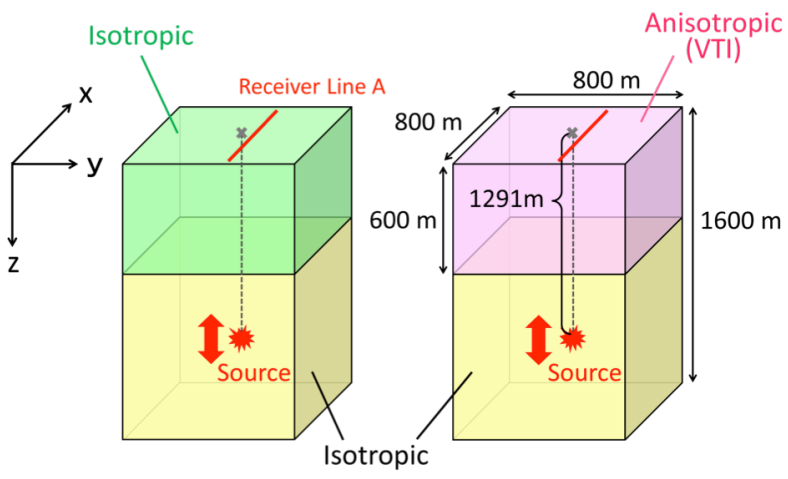

(a) Isotropic anomaly model

(b) Anisotropic anomaly model

Figure 1 Isotropic anomaly model (a) has an isotropic top medium and anisotropic anomaly model (b) has an anisotropic (VTI) top medium.

Table 1 Vertical phase velocities and density of the isotropic and anisotropic anomaly models

\begin{tabular}{|cccc|}
\hline & $\begin{array}{c}\text { P-wave } \\
\text { Vertical Velocity } \\
\mathbf{V}_{\mathbf{p}-\mathbf{z}}(\mathbf{m} / \mathbf{s})\end{array}$ & $\begin{array}{c}\text { S-wave } \\
\text { Vertical Velocity } \\
\mathbf{V}_{\mathbf{s - \mathbf { z }}}(\mathbf{m} / \mathbf{s})\end{array}$ & $\begin{array}{c}\text { Density } \\
\boldsymbol{\rho}\left(\mathbf{k g} / \mathbf{m}^{3}\right)\end{array}$ \\
\hline Top layer & 2200 & 1400 & 2500 \\
\hline Bottom layer & 3200 & 1900 & 2500 \\
\hline
\end{tabular}




\section{RESULTS AND DISCUSSION}

Fig. 2 shows z-component of the particle velocity observed at receiver line A in Fig. 1. The line $\mathrm{A}$ is in $\mathrm{y}=3.125 \mathrm{~m}$ and offset is from $\mathrm{x}=$ $-803.25 \mathrm{~m}$ to $\mathrm{x}=803.25 \mathrm{~m}$. Fig. 2 (a) is the seismic data of the isotropic anomaly model and Fig. 2 (b) is that of the anisotropic anomaly model. Red, green and blue colored zones represent time zone $\mathrm{t}=0.6-0.8 \mathrm{sec}, \mathrm{t}=0.8-1.0 \mathrm{sec}$ and $\mathrm{t}=1.0-1.2$ sec, respectively. In both Fig. 2 (a) and (b), initial $\mathrm{P}$-waves are observed around $\mathrm{t}=0.5 \mathrm{sec}$ and initial $\mathrm{S}$-waves are observed around $\mathrm{t}=0.8 \mathrm{sec}$. Then, waves observed between initial $\mathrm{P}$-waves and initial S-waves are mainly converted P-S waves and multiple reflection waves.

To see how strong anisotropy of media has influence on the wavefield, we conducted the orbital analysis using seismic data. This analysis is valid for estimating the kinds of seismic wave from direction and amount of the particle displacement. Fig. 3 (a) and (b) show the orbits in XZ-plane of two receiver points on the receiver line $\mathrm{A}$ of the isotropic anomaly model, and Fig. 3 (c) and (d) are those of the anisotropic anomaly model. Fig. 3 (a), (c) and (e) are orbits of offset $x=-403.125 \mathrm{~m}$, and Fig. 3 (b), (d) and (f) are orbits of $x=-153.125 \mathrm{~m}$. Colors of orbit lines correspond to the colored time zones in Fig. 2. In Fig. 3, we can see the difference between Fig. 3 (a) and (c) in time 0.6-0.8 sec, the arrival time between initial $\mathrm{P}$-wave and initial $\mathrm{S}$-wave. The results of orbital analysis show that the waveforms appear in $0.6-0.8 \mathrm{sec}$ are due to P-S converted waves, and that the influence of anisotropy appears in P-S converted waves is relatively strong comparing to initial $\mathrm{P}$-wave and initial S-wave. In general anisotropic medium, wave vibration direction of $\mathrm{P}$-wave is not completely parallel to propagation direction and slightly contains shear deformation. So, in the boundary between top media and bottom media in our models, shear waves are generated from P-P transmitted waves, and these shear waves are observed as large amplitude of P-S converted waves in Fig. 3 (c) and (d).

Fig. 3 (e) and (f) is the result of orbital analysis using weak anisotropic anomaly model with the degree of anisotropy $10 \%$. Fig. 3 (c) and (e) indicate that degree of anisotropy has influence on amplitude of P-S converted waves. Our results strongly indicate that one of the causes of shear wave generation could be the anisotropic nature of subsurface materials where seismic waves travel through.

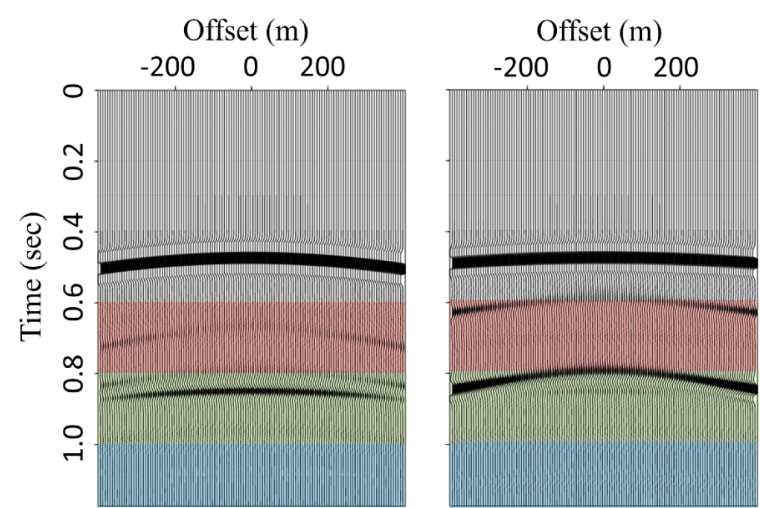

(a) Isotropic anomaly model (b) Anisotropic anomaly model

Figure 2 Seismic data of $\mathrm{z}$-component of isotropic anomaly model and anisotropic anomaly model received on receiver lines (Line A).

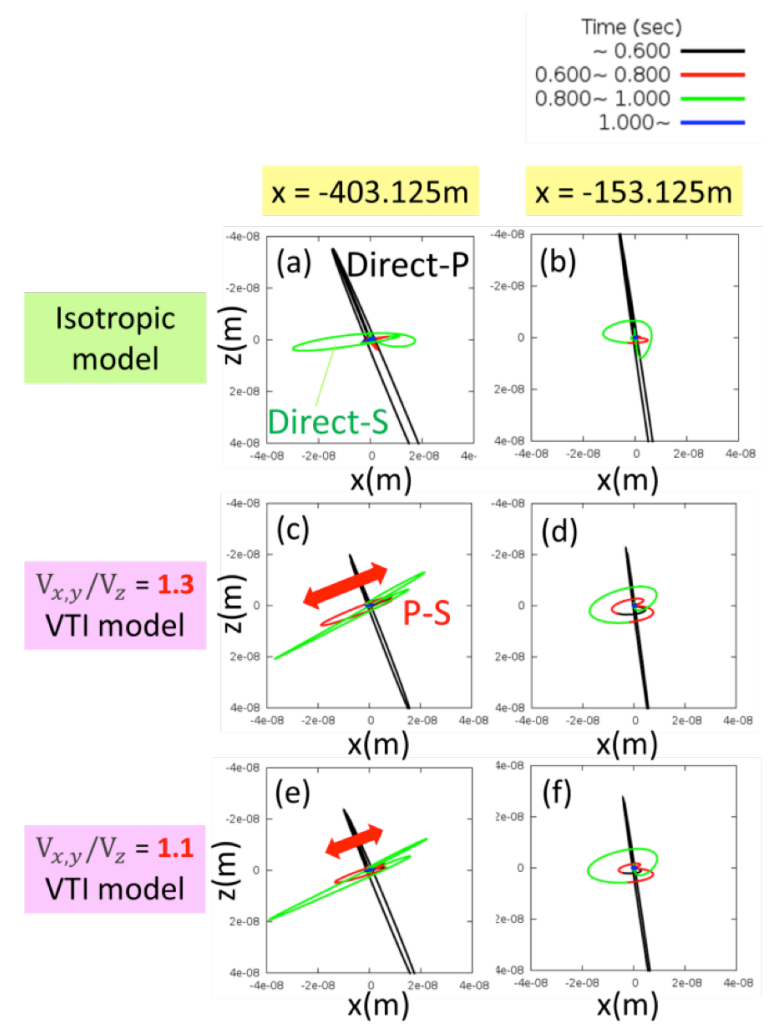

Figure 3 Orbits in XZ-plane of isotropic anomaly model (a) (b), anisotropic anomaly model (c) (d) and weak anisotropic VTI model (e) (f). Left ones (a) (c) (e) are particle motions in receiver points $\mathrm{x}=-403.125 \mathrm{~m}$, and right ones (b) (d) (f) are particle motions in receiver points $\mathrm{x}=-153.125 \mathrm{~m}$. 


\section{CONCLUSION}

In this study, we simulate seismic wave propagation in anisotropic and isotropic media to investigate the influence of anisotropy on received waveforms, especially on P-S converted waves. By using 3-D layered structure models, we compare the seismic data and orbits between isotropic and anisotropic anomaly models.

$\mathrm{P}-\mathrm{S}$ converted wave, observed in time between initial P-wave and S-wave, has effective information about anisotropic parameters, and that we could obtain information about near surface anisotropy from P-S converted waves. It is necessary to investigate further the relation between P-S converted waves and underground anisotropy.

\section{REFERENCES}

1) Sone, H., 2012, Mechanical properties of shale gas reservoir rocks and its relation to the in-situ stress variation in shale gas reservoir, Ph.D. Thesis, Stanford University, 225pp.

2) Thomsen, L., 1986, Weak elastic anisotropy, Geophysics, 51 (10), 1954-1966.

3) Alkhalifah, T., 2000, An acoustic wave equation for anisotropic media, Geophysics, 65 (4), 1239-1250.

4) Matsumoto, S., 2009, A review on structure of the earth using scattered seismic waves by heterogeneity, Jishin, 61, 209-216.

5) Aki, K. and Richards, P. G., 2002, Quantitative seismology, University Sience Book (transrated in Japanese by Uenishi, K., Kame, N., Aochi, H., 2004, Kokon-shoin)

6) Virieux, J., 1986, P-SV wave propagation in heterogeneous media: Velocity-stress finite-difference method, Geophysics, $\mathbf{5 1}$ (4), 889-901.

7) Saenger, E. H., Gold, N., and Shapiro, S. A., 2000, Modeling the propagation of elastic waves using a modified fnite-difference grid, Wave motion, 31 (1), 77-92.

8) Saenger, E. H., and Bohlen, T., 2004, Finite-difference modeling of viscoelastic and anisotropic wave propagation using the rotated staggered grid, Geophysics, 69 (2), 583-591.

9) Komatitsch, D., Martin, R., 2007, An unsplit convolutional perfectly matched layer improved at grazing incidence for the seismic wave equation, Geophysics, 72, 155-167. 